\title{
Intervenções cognitivo comportamentais para gerenciamento de estresse em pacientes oncológicos: uma revisão
} sistemática*

\section{Cognitive behavioral interventions for stress management in oncology patients: a systematic review}

Cristiano Pereira de Oliveira ${ }^{1}$ Marcela Alves de Moraes ${ }^{2}$ Tayse Conter de Moura ${ }^{3}$
Recebido em: 09/03/2016.

Aprovado em: 02/12/2016.

Mestre em Psicologia pela Pontifícia Universidade Católica do RS (PUCRS), Especialista em Terapias Cognitivo-Comportamentais e Psico-Oncologia. Instituições: CliniOnco e FADERGS. Contatos: deoliveira1982@gmail. com, Porto Alegre, Rio Grande do Sul, Brasil. Correspondências sobre o manuscrito: Rua Gonçalves Dias, 54 Apto 305 CEP: 90130-060; telefone: 5551 9159-9963.

2 Acadêmica em Psicologia pela PUCRS; CliniOnco Porto Alegre; marcela.alves.moraes@ gmail.com; Porto Alegre, Rio Grande do Sul, Brasil. Trabalhou no método e na revisão, organização final do estudo e submissão deste.

3 Acadêmica em Psicologia pela PUCRS; CliniOnco Porto Alegre; tayse.conter@gmail.com; Porto Alegre, Rio Grande do Sul, Brasil. Trabalhou em parte na revisão da literatura e organização final do estudo.

\section{Resumo}

O tratamento oncológico gera altos índices de estresse que podem causar potenciais prejuízos na vida dos pacientes, além de possíveis efeitos deletérios em variáveis biológicas relacionadas ao tratamento. O objetivo do presente estudo foi investigar quais técnicas da Terapia Cognitivo Comportamental (TCC) são, empiricamente, testadas nesse contexto. Para tal, a metodologia empregada foi a de Revisão sistemática de artigos no período de 2010 a 2015, sendo utilizadas as bases de dados: Pubmed, PsychInfo, Omnis, Scholar Google. A qualidade dos artigos foi avaliada conforme escala de JADAD por dois examinadores independentes. Os resultados obtidos revelaram que, dentro das técnicas da (TCC), as intervenções baseadas em Mindfullness, o protocolo biocomportamental e o relaxamento foram as mais eficazes e empiricamente testadas para gerenciamento de estresse em pacientes oncológicos.

Palavras-chave: Gerenciamento de estresse. Câncer. Psicoterapia Cognitivo-Comportamental.

\begin{abstract}
The cancer treatment generates high levels of stress that can cause potential damage in the lives of patients as well as possible deleterious effects on biological variables related to treatment. The aim of this study was to investigate what techniques of Cognitive Behavioral Therapy (CBT) are empirically tested in this context. To this end, the methodology used was the systematic review of articles in the period 2010 to 2015, the databases being used: Pubmed, PsychInfo, Omnis, Google Scholar. The quality of the articles was assessed as JADAD scale by two independent examiners. The results were that within the techniques (CBT) interventions based on Mindfullness, biobehavioural protocol and relaxation were the most effective and empirically tested for stress management in cancer patients. Keywords: Stress management. Cancer. Cognitive Behavioral Therapy.
\end{abstract}




\section{Introdução}

O Câncer é considerado um problema de saúde pública, figurando entre as principais causas de morte no mundo e a $3^{\text {a }}$ causa no Brasil. São esperados 596.000 novos casos para 2016 e 2017, segundo estimativas do Instituto Nacional do Câncer (INCA, 2016). Os tratamentos para o câncer podem compreender intervenção cirúrgica, quimioterapia (QT), radioterapia (RDT) e, em alguns casos, o uso de anticorpos monoclonais. Esses tratamentos podem ocorrer de forma separada, conjunta ou continuada (SBOC, 2011). Ainda que haja exceções, apenas ao final da década de 70 estudos sobre os impactos e possíveis intervenções psicológicas ao paciente oncológico começaram a surgir na quantidade e qualidade necessária para atender à demanda crescente. Os resultados desses trabalhos nos permitem a compreensão e ação nos processos comportamentais e psicológicos da adaptação à doença visando à qualidade de vida (ANDERSEN et al., 1994). Adultos que sofrem de estresse crônico podem ter prejuízos biológicos importantes e, muitas vezes ter dificuldades de se adaptar à realidade conturbada que o diagnóstico de câncer e tratamento podem requerer. Essa condição estressora pode afetar o funcionamento imunológico reduzindo a capacidade dos indivíduos de resistir à doença e aumentando, dessa forma, a probabilidade do surgimento de metástases (ANDERSEN et al., 1998). Os impactos do estresse do diagnóstico e do tratamento resultam em potenciais prejuízos à imunocompetência dos pacientes e isso pode estar relacionado, apesar de estudos insuficientes e contraditórios, com progressão de doença e mortalidade. A resposta ao estresse é caracterizada como provável mediadora dos efeitos psicossociais do estresse na progressão do câncer. Esse processo envolve a ativação do sistema nervoso autônomo e do eixo hipotálamo-hipófise-adrenal (HPA). Há evidências de que existe variação conforme o desenvolvimento da doença, despontando como período crítico o período pericirúrgico com impactos deletérios do estresse (LUGTENDORF et al., 2010; GARSSEN et al., 2010).Ainda que haja lacunas sobre a relação entre hormônios do estresse e sintomas depressivos em pacientes oncológicos, um estudo com 227 mulheres com neoplasia mamária, em estadiamentos II e III, indicou relação negativa entre sintomas depressivos e cortisol no diagnóstico, porém com uma mudança de quadro a partir da cirurgia. Dessa sintomas depressivos, mas alterar essa relação ao longo do tempo (SALENE et al., 2014).

$\mathrm{Na}$ última década, vários estudos têm buscado responder a função da regulação neuroendócrina no desenvolvimento e progressão do câncer. Necessariamente, os efeitos da ativação do sistema nervoso simpático resultam em resposta celular imune, processos inflamatórios, angiogênese, invasão tumoral e crescimento tumoral (ARMAIZ-PENA et al., 2013).As evidências sugerem que os fatores comportamentais podem modular a resposta ao estresse e com isso tem função capital na progressão do câncer e desenvolvimento de metástases. Além disso, afetam a qualidade de vida, por isso, intervenções comportamentais que visem alterar as rotas neuroendócrinas devem ser objetivo das equipes de atenção oncológica (CONTANZO et al., 2011). As intervenções para manejo de estresse baseadas em intervenções psicossociais não demonstraram ser eficazes para manejar os estressores do tratamento conforme evidenciou estudo com mulheres com câncer de mama em quimioterapia adjuvante. Esse estudo sugere o emprego de outras intervenções mais diretivas do que a utilizada, como Tai Chi, grupos de expressão e crescimento espiritual (ROBINS et al., 2013).Nesse sentido, a avaliação da efetividade de uma intervenção combinada de técnicas biocomportamentais e terapia cognitiva comportamental, em pacientes oncológicos deprimidos, sendo estes acompanhados e psicoeducados a manejar o estresse do tratamento, obteve como resultados uma melhora significativa em sintomas depressivos. 19 dos 21 pacientes que completaram o estudo apresentaram critérios para alta (da depressão). Além disso, houve uma melhora significativa de saúde e fadiga mental. Bem como, houve uma diminuição da ansiedade e dos níveis de estresse associados ao câncer (BROTHERS et al., 2011).Há evidências consistentes indicando que a cronicidade de fatores estressores e a ansiedade apresentam relação com perfis de transcrição pró-inflamatórios e leucócitos. Buscando analisar o impacto de intervenções cognitivo-comportamentais, 199 mulheres com câncer de mama em estadiamento I-III foram randomizadas para um protocolo específico de 10 semanas e grupo controle. Os achados indicaram que as mulheres em adjuvância para câncer de mama, que passaram pelo protocolo específico, obtiveram uma reversão da expressão pró-inflamatória. Logo, isto indica que intervenções cognitivo comportamentais podem influenciar a saúde física positivamente. Além disso, essa mesma intervenção 
demonstrou-se útil no combate de insônia e fadiga, bem como diminuição dos níveis de ansiedade nas pacientes com câncer de mama em adjuvância (ANTONI et al., 2013; VARGAS et al., 2013).

No estudo de Antoni et al. (2001), foram testados os efeitos de uma intervenção de gestão do stress de dez semanas de grupo cognitivo-comportamental entre 100 mulheres recém-tratadas para câncer de mama nos estadiamentos O-II. A intervenção reduziu a prevalência de depressão moderada (que se manteve relativamente estável na condição de controle), mas não afetou outras medidas de sofrimento emocional. A intervenção, também, aumentou relatos das participantes que ter câncer de mama tinham feito contribuições positivas para suas vidas, e aumentou o otimismo generalizado. Ambos permaneceram, significativamente, elevados em 3 meses de acompanhamento da intervenção. Uma análise posterior revelou que a intervenção teve seu maior impacto sobre essas 2 variáveis entre as mulheres que estavam em menor otimismo no início do estudo.

Em pacientes com câncer de mama em estágio inicial, uma intervenção de 10 semanas reverteu-se em regulação positiva relacionada com ansiedade além de ter uma ação inibitória da expressão de genes pró-inflamatória em leucócitos circulantes. Esses resultados indicam que intervenções cognitivo-comportamentais têm impactos em regulação emocional que resultam em saúde física e, além disso, pode ter potencial para alterar processos inflamatórios que afetam tanto questões afetivas quanto cognitivas do cérebro (ANTONI et al., 2012).A mesma técnica de intervenção foi utilizada em pacientes com câncer de próstata e os resultados, também, indicam melhorias nesses mesmos fatores dos encontrados com os estudos com câncer de mama. Porém, como diferencial, buscou-se avaliar o funcionamento sexual, o que se mostrou com comportamento independente. Além disso, ficou evidenciado que questões que envolvem cognições acerca da doença são uma necessidade de atenção nesse contexto (TRAEGER et al., 2013).Outra prática que começou a ficar bastante difundida a partir da década de 80 , que começou a integrar a medicina comportamental por meio dos programas de Jon Kabat-Zinn e que tinha como um dos principais objetivos a redução do estresse foi a prática de mindfulness. O termo mindfulness, também, é definido como "atenção plena", concentração no aqui e no agora de forma atencional e sem julgamento. A prática de mindfulness veio para trabalhar na contramão do fun- cionamento do que o autor chama de "piloto automático", e sim fazer as pessoas se conectarem de maneira intencional com o seu momento presente (VANDENBERGHE; SOUSA, 2006).

Ao analisar o uso da terapia cognitiva baseada em Mindfulness para alivio de sofrimentos psicológicos em pacientes oncológicos, uma vez que o diagnóstico oncológico não tem repercussões, exclusivamente, físicas, como também psicológicas com impactos na qualidade de vida desses pacientes. Os resultados indicaram que técnicas cognitivas baseadas em Mindfulness apresentam significativa segurança e eficácia para gerenciamento do estresse, além de diversos problemas tais como dor, fadiga, náusea e vomito. Por fim aponta para a sugestão de atenção integral e multiprofissional como sugestão para o cuidado de pacientes oncológicos (ABDOLHAHI et al., 2014).O objetivo deste estudo foi avaliar os resultados de ensaios clínicos randomizados realizados na população oncológica com intervenções cognitivas e comportamentais para gestão de estresse e suas respostas empiricamente testadas nesse contexto.

\section{Metodologia}

Foram feitas buscas sistemáticas de ensaios clínicos randomizados de intervenções cognitivo comportamentais para gestão de estresse em pacientes oncológicos. Foi adotada a perspectiva metodológica preconizada para revisão sistemática (BRECKWELL et al., 2013).

As buscas foram realizadas em bases eletrônicas, sendo considerado o período compreendido entre 2010 e 2015. Foram consultadas as seguintes bases de dados: Pubmed, PsychInfo, Google Scholar e Sistema de pesquisa múltipla Omnis da PUCRS. As buscas seguiram os seguintes critérios de busca (Figura 1) adotados após a consulta ao Medical Subject Headings (MESH). Termo de buscas que foram utilizados após a consulta ao Mesh: Cancer; Stress Management; Cognitive Behavioral Therapy; CBT; Intervention; No Pubmed foram ainda adotados os filtros: Clinical Trials, cinco anos e humanos; No PsychInfo foram adicionadas a solicitação de que Cancer e Stress Management estivessem nas palavras chaves dos artigos pesquisados. No Google Scholar, foi adotado o filtro com a palavra Randomized Controled Trial, além de que contasse com as palavras Intervention, Cognitive Behavioral Therapy and Cancer. Os critérios de inclusão constituíram-se de analisar, apenas, ensaios clínicos 
randomizados de intervenções cognitivo comportamentais para gerenciamento de estresse em pacientes oncológicos. Estudos que avaliavam aspectos metodológicos, tais como validação de instrumentos ou que envolviam intervenções não exclusivamente psicoterápicas foram excluídos.

A extração dos dados se conduziu de forma independente por dois examinadores e foi utilizada a escala Jadad para avaliação da qualidade dos estudos (JADAD et al., 1996).

A Escala de qualidade de ensaios clínicos Jadad é um instrumento de avaliação da qualidade de ensaios clínicos aleatórios que foi desenvolvida por Alejandro Jadad-Bechara em seus estudos sobre a qualidade e utilidade desta modalidade de pesquisa para o avanço científico. Ele classificou os estudos entre 0 e 5 quanto a suas qualidades metodológicas sendo que 0 são considerados de qualidade muito pobre e 5 excelente. Considera-se, para fins de avaliação que apresentam má qualidade, todos estudos que apresentem escores $\leq 3$ (JADAD et al., 1996).

\section{Resultados}

A figura 1 mostra o fluxograma de estudos encon- trados segundo os critérios de inclusão supracitados. Ao todo, 686 artigos foram identificados, sendo a maioria excluída pela simples análise de compatibilidade frente aos critérios adotados. Dos sete artigos que satisfizeram os critérios estabelecidos obtivemos duplicidades de resultados em dois deles Pubmed e Psychinfo e Pubmed e Omnis.

Figura 1. Número de artigos encontrados e selecionados segundo critérios

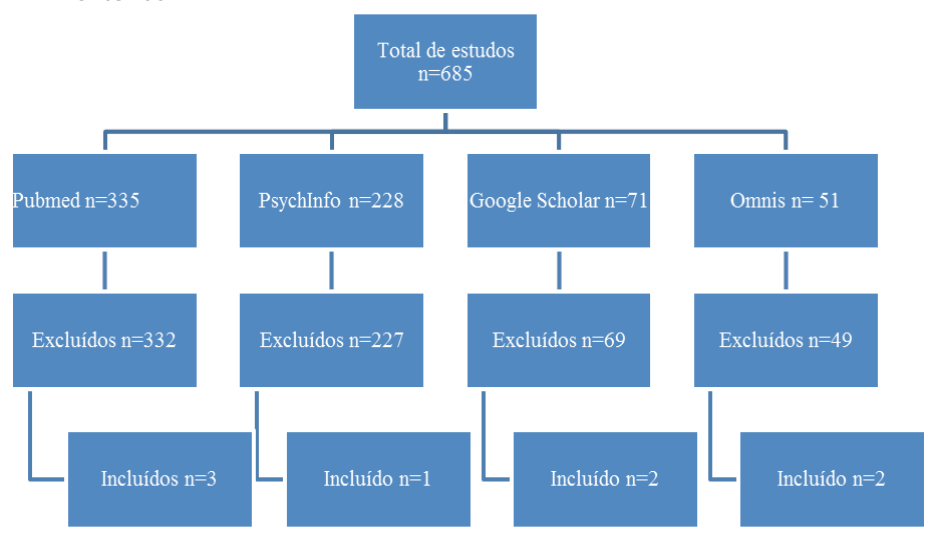

Fonte: Dos autores dados dos artigos encontrados na revisão, tais como escore Jadad, caracterização, instrumentos de avaliação e resultados mais relevantes.

Quadro 1. Resumo de estudos

\begin{tabular}{|c|c|c|c|c|c|c|}
\hline Autor, Ano, País & Amostra & Grupo Intervenção & Grupo Controle & Intrumentos utilizados & Resultados & JADAD \\
\hline $\begin{array}{l}\text { Stagl et al., } 2014 \\
\text { Estados Unidos }\end{array}$ & $\begin{array}{l}\mathrm{n}=130 \\
\text { Pacientes com câncer } \\
\text { de mama }\end{array}$ & $\begin{array}{l}\mathrm{n}=70 \\
10 \text { semanas de terapia } \\
\text { cognitivo comportamental } \\
\text { para gestão de estresse }\end{array}$ & $\begin{array}{l}n=60 \\
1 \text { dia de grupo de } \\
\text { psicoeducação }\end{array}$ & $\begin{array}{l}\text { Escala de Depressão de } \\
\text { Hamilton no baseline; } \\
\text { Escala para depressão } \\
\text { do Centro de Estudos } \\
\text { Epidemiológicos (CES-D) no } \\
\text { follow up; Questionário sócio } \\
\text { demográfico (no baseline e } \\
\text { folow-up) }\end{array}$ & $\begin{array}{l}0 \text { grupo que participou de } \\
\text { TCC para gestão de estresse } \\
\text { apresentou, significativamente, } \\
\text { menos sintomas depressivos } \\
(\mathrm{M}=9,99 \text { e } \mathrm{dp}=0,93) \text { do que } 0 \\
\text { Controle }(\mathrm{M}=12,97 \mathrm{dp}=0,99) \\
\text { nível de signficância de } 0,30 \\
\text { ajustado pelos fatores de co- } \\
\text { variância } \mathrm{p}=0,12\end{array}$ & 3 \\
\hline $\begin{array}{l}\text { Lechner et al., } \\
\text { 2014, Estados } \\
\text { Unidos }\end{array}$ & $\begin{array}{l}\mathrm{n}=114 ; \mathrm{I}=57 \\
\text { mulheres negras } \\
\text { com câncer de } \\
\text { mama em quaisquer } \\
\text { estadiamentos que } \\
\text { tenham concluído } \\
\text { tratamentos há pelo } \\
\text { menos um ano. }\end{array}$ & $\begin{array}{l}\mathrm{n}=57 \\
\text { IM: } 50,16(7,89) \\
10 \text { sessões semanais } \\
\text { de terapia cognitivo } \\
\text { comportamental em grupo. }\end{array}$ & $\begin{array}{l}\mathrm{n}=57 \\
\text { IM: } 52,07(9,93) \\
\text { Um encontro } \\
\text { educacional não } \\
\text { especifico para } \\
\text { questões importantes } \\
\text { dos sobreviventes }\end{array}$ & $\begin{array}{l}\text { Questionário sócio } \\
\text { demográfico;Qualidade de } \\
\text { Vida (Facit-B); Humor (POMS- } \\
\text { SV); Pensamentos Intrusivos } \\
\text { (IES-R-Int); Sintomas } \\
\text { depressivos (CES-D); Estresse } \\
\text { percebido (PSS);No baseline, } \\
\text { pós intervenção e follow up } 6 \\
\text { meses após; }\end{array}$ & $\begin{array}{l}\text { Ambos os grupos estudados } \\
\text { apresentaram melhoras } \\
\text { significativas em todas as } \\
\text { avaliações realizadas. Os } \\
\text { resultados sugerem que as } \\
\text { melhorias pode ter se dado } \\
\text { devido a um treinamento } \\
\text { intensivo de manejo de estresse, } \\
\text { ampla oferta de informação no } \\
\text { grupo de psicoeducação para } 0 \\
\text { câncer de mama. }\end{array}$ & 3 \\
\hline
\end{tabular}




\begin{tabular}{|c|c|c|c|c|c|c|}
\hline $\begin{array}{l}\text { Henderson et al, } \\
\text { 2013, Estados } \\
\text { Unidos }\end{array}$ & $\begin{array}{l}\mathrm{n}=172 \\
\text { mulheres com câncer } \\
\text { de mama cujo } \mathrm{n}=77 \\
\text { fizeram RDT antes } \\
\text { do estudo e } \mathrm{n}=40 \text {, } \\
\text { durante. } \\
\text { Ml: } 50(8)\end{array}$ & $\begin{array}{l}\mathrm{n}=53 \\
8 \text { semanas de Mindfullness } \\
\text { para gestão de estresse } \\
\text { semanal em grupos }\end{array}$ & $\begin{array}{l}\mathrm{n}=58 \\
8 \text { semanas baseado na } \\
\text { educação nutricional, } \\
\text { sob a perspectiva da } \\
\text { teoria social cognitiva }\end{array}$ & $\begin{array}{l}\text { Questionário sócio } \\
\text { demográfico; DWI (Dealing } \\
\text { With Illness questionaire); } \\
\text { Fact-B (Breast Cancer version } \\
\text { of Functional Assesment of } \\
\text { Cancer Therapy) adicionado } \\
\text { itens de espiritualidade; } \\
\text { SOC (Sense de Coherence } \\
\text { Scale);MMAC (Mini-mental } \\
\text { Adjustment to Cancer Scale); } \\
\text { SCL (Symptom Checklist-90 } \\
\text { revised); CEC (Courtauld } \\
\text { Emotional Control Scale) }\end{array}$ & $\begin{array}{l}0 \text { uso de Mindfullness em } \\
\text { mulheres em radioterapia } \\
\text { demonstrou melhor } \\
\text { significativamente } 16 \text { variáveis } \\
\text { psicossociais em comparação } \\
\text { com os controles. } 0 \text { estudo } \\
\text { evidenciou que a técnica pode } \\
\text { facilitar } 0 \text { ajustamento de } \\
\text { pacientes durante o tratamento } \\
\text { oncológico. }\end{array}$ & 3 \\
\hline Traeger et al., 2013 & $\begin{array}{l}\mathrm{n}=237 \text { homens com } \\
\text { câncer de próstata } \\
\text { estadiamentos I ou } \\
\text { II diagnosticado nos } \\
\text { últimos } 18 \text { meses }\end{array}$ & $\begin{array}{l}\mathrm{n}=148 \\
\text { Intervençöes cognitivo } \\
\text { comportamentais para } \\
\text { manejo e estresse }\end{array}$ & $\begin{array}{l}\mathrm{n}=109 \\
\text { Não receberam as } \\
\text { intervenções cognitivo } \\
\text { comportamentais }\end{array}$ & $\begin{array}{l}\text { FACT-G (Functional } \\
\text { Assessment of Cancer } \\
\text { Therapy-General Module), } \\
\text { PPS(Perceived Stress Scale), } \\
\text { IPQ-R (IIIness Perception } \\
\text { Questionnaire), EPIC } \\
\text { (Expanded Prostate Cancer } \\
\text { Index Composite) }\end{array}$ & $\begin{array}{l}\text { Após receberem intervenções } \\
\text { da TCC pra ME, os pacientes } \\
\text { demonstraram melhora } \\
\text { significativa no bem-estar } \\
\text { emocional quando comparados } \\
\text { ao grupo controle. Disfunções } \\
\text { sexuais e urinária não } \\
\text { influenciaram os ganhos obtidos } \\
\text { com as intervenções de TCC }\end{array}$ & 5 \\
\hline $\begin{array}{l}\text { Groarke et al., } \\
2013\end{array}$ & $\begin{array}{l}\mathrm{n}=355 \\
\text { Estudo longitudinal } \\
\text { randomizado } \\
\text { controlado com } \\
\text { pacientes com câncer } \\
\text { de mama em fase } \\
\text { de quimioterapia } \\
\text { adjuvante com follow } \\
\text { up após } 12 \text { meses. }\end{array}$ & $\begin{array}{l}\mathrm{n}=177 \\
\text { Recebeu terapia cognitivo } \\
\text { comportamental baseada na } \\
\text { redução de estresse durante } \\
5 \text { semanas por } 3 \text { horas } \\
\text { por dia. }\end{array}$ & $\begin{array}{l}\mathrm{n}=178 \\
\text { Recebeu tratamento } \\
\text { padrão durante 9-20 } \\
\text { semanas }\end{array}$ & $\begin{array}{l}\text { Questionário que avalia } \\
\text { stress global (Global } \\
\text { Stress);Questionário específico } \\
\text { de stress do câncer (Cancer- } \\
\text { specific-stress);HADS - para } \\
\text { avaliar o níveis de ansiedade } \\
\text { e depressáo;Benefit Finding } \\
\text { - que indica os benefícios } \\
\text { encontrados a partir das } \\
\text { intervençōes utilizadas entre } \\
\text { o grupo controle e o de } \\
\text { intervenção. }\end{array}$ & $\begin{array}{l}\text { As análises de variância } \\
\text { revelaram que os pacientes } \\
\text { que receberam intervenção } \\
\text { (CBMS) tiveram uma redução } \\
\text { significativa no estresse global } \\
\text { e na ansiedade comparado ao } \\
\text { grupo controle. Contudo, essas } \\
\text { diferenças não foram mantidas } \\
\text { no follow up de } 12 \text { meses }\end{array}$ & 4 \\
\hline $\begin{array}{l}\text { Lloyd-Willians et } \\
\text { al.,2013 }\end{array}$ & $\begin{array}{l}\mathrm{n}=100 \\
\text { Ml: } 66 \text { anos; } 68 \% \\
\text { mulheres; todos } \\
\text { diagnosticados com } \\
\text { câncer nos últimos } 12 \\
\text { meses e possuindo } \\
\text { ampla gama de } \\
\text { diagnóstico. }\end{array}$ & $\begin{array}{l}\mathrm{n}=49 \\
\text { participaram de entrevista } \\
\text { narrativa focada, sendo } \\
\text { encorajados a falar de } \\
\text { aspectos biopsicossociais } \\
\text { referentes à doença. As } \\
\text { entrevistas foram gravadas } \\
\text { para garantir o rigor e } \\
\text { qualidade das intervençōes. }\end{array}$ & $\begin{array}{l}\mathrm{n}=51 \\
\text { receberam tratamento } \\
\text { standard }\end{array}$ & $\begin{array}{l}\text { Numerical Visual analogue } \\
\text { scale of suffering, the six item } \\
\text { Brief Edinburgh Depression } \\
\text { Scale (BEDS), FACIT } \\
\text { Spirituality questionnaire } \\
\text { and Edmonton Symptom } \\
\text { Assessment Scale (ESAS) }\end{array}$ & $\begin{array}{l}\text { Não houve diferença significativa } \\
\text { na comparação entre os dois } \\
\text { grupos após a realização dos } \\
\text { respectivos procedimentos. } \\
\text { Durante a realização dos } \\
\text { estudos, } 25 \text { pacientes faleceram. } \\
\text { Sintomas de fadiga, sonolência e } \\
\text { perda de apetite foram maiores } \\
\text { no grupo de intervenção, } \\
\text { indicando que pacientes } \\
\text { randomizados para esse grupo } \\
\text { estavam em estado global de } \\
\text { saúde pior }\end{array}$ & 3 \\
\hline $\begin{array}{l}\text { Gudenkauf et al., } \\
2015 .\end{array}$ & $\begin{array}{l}\mathrm{n}=183 \text { mulheres de } \\
\text { estadiamento } 0 \text {-III }\end{array}$ & $\begin{array}{l}\text { (1) grupo de relaxamento } \\
\text { de } 5 \text { semanas (2) grupo } \\
\text { de terapia cognitivo- } \\
\text { comportamental para } \\
\text { redução de estresse }\end{array}$ & $\begin{array}{l}\text { de } 5 \text { semanas na qual } \\
\text { foram abordadas } \\
\text { questões educacionais } \\
\text { sobre câncer de mama } \\
\text { e saúde. }\end{array}$ & $\begin{array}{l}\text { Affects Balance Scale para } \\
\text { avaliação de depressão } \\
\text { (DEROGATIS, 1975); Sub- } \\
\text { escala de pensamentos } \\
\text { intrusivos (IES-I) do Impact of } \\
\text { Event Scale - Revised (WEISS, } \\
\text { 2007); Sickness Impact } \\
\text { Profile-Social Interaction } \\
\text { subscale (BERGNER et al., } \\
\text { 1981); Functional Assessment } \\
\text { of Cancer Therapy-Breast } \\
\text { (BRADY et al., 1997); Social } \\
\text { Providions Scale (TRONA; } \\
\text { RUSSEL, 1987) }\end{array}$ & $\begin{array}{l}\text { 0s dois grupos de intervenção } \\
\text { tiveram depressão diminuída. } \\
\text { Grupo de TCC maior bem-estar } \\
\text { emocional e qualidade de vida } \\
\text { relatada, bem como menor } \\
\text { intrusão. Grupo de Relaxamento } \\
\text { apresentou melhora em } \\
\text { questões sociais e grande } \\
\text { índice de confiança na técnica } \\
\text { apresentada. }\end{array}$ & 4 \\
\hline
\end{tabular}




\section{Discussão}

Os achados indicam que há eficácia e benefício no uso de intervenções cognitivo comportamentais para gerenciamento de estresse. As evidências indicam que os benefícios extrapolam para além de questões emocionais tendo impactos diretos em aspectos biológicos e físicos, interferindo em processos como progressão e mortalidade (CONSTANZO et al., 2011). As intervenções biocomportamentais são divididas em dois protocolos clínicos, um com 26 sessões individuais ou grupais desenvolvido pela Dra. Barbara Andersen e colaboradores da Ohio State University e o modelo de intervenção grupal do Dr. Michel Antoni e colaboradores da University of Miami. Considerando-se os critérios de pesquisa e os artigos encontrados, foram incluídos nesta revisão, apenas, estudos do protocolo do Dr. Antoni (ANDERSEN et al., 2009; ANTONI et al., 2010).

O modelo biocomportamental é o modelo pelo qual as intervenções são pensadas, ou seja, as intervenções foram planejadas em forma de protocolos que incluem um arsenal de intervenções cognitivas e comportamentais, as quais objetivam minorar os efeitos dos estressores. Nesse sentido, o protocolo torna-se manualizado e permite aos terapeutas a uniformização de condutas clínicas. A partir desse modelo de entendimento, as intervenções são direcionadas a intervenções para manejo de aspectos sociais (comunicação assertiva, rede de apoio, resolução de problemas) aspectos emocionais (ansiedade) e biológicos (com estimulação para adoção de hábitos alimentares e adoção de atividades físicas) (ANTONI et al., 2012; ARMAIZ-PENA et al., 2013).

Um estudo foi descrito Mindfulness para gerenciamento de estresse, o qual se demonstrou eficaz na redução do estresse com impacto positivo considerável. Com a aprendizagem pelos pacientes de atenção plena no presente sem a consciência crítica e em protocolo de 05-08 semanas, consegue-se modificar a capacidade de avaliação de situações estressantes, reduzindo significativamente a excitação psicofisiológica. Além disso, essa técnica se demonstrou útil para combater a insônia em pacientes demonstrando eficácia comparada a psicofármacos (HENDERSON et al., 2013; GARLAND et al., 2014).Quanto à avaliação da qualidade dos ensaios clínicos, todos os estudos encontrados foram classificados segundo a escala Jadad, como com qualidade maior ou mínima para serem caracterizados como tal (JADAD et al., 1996).As intervenções cognitivas comportamentais, portanto, apresentaram-se como recursos seguros para uso em pacientes com câncer de mama, tanto a baseada em Mindfulness quanto protocolos fundamentados nas técnicas de manejo fundamentadas no modelo biocomportamental. Nesse sentido, fica como lacuna a comprovação empírica em outros diagnósticos oncológicos, necessitando novos estudos para avaliar a eficácia desses modelos para os demais diagnósticos.

\section{Considerações finais}

As intervenções cognitivas e comportamentais, portanto, apresentaram-se como recursos seguros e efetivos para uso em pacientes com câncer de mama, tanto a baseada em Mindfulness quanto protocolos fundamentados nas técnicas de manejo fundamentadas no modelo biocomportamental. Apesar das evidências se restringirem a pacientes com câncer de mama, podemos, salvaguardadas as especificidades dessa condição clínica, pensar na utilização dessas técnicas como promotoras de saúde tanto física, quanto mental e como recurso garantidor de qualidade de vida em pacientes oncológicos. Ainda que carecedora de evidências para a utilização precisa em outros diagnósticos o gerenciamento de estresse se mostra promissor como adjuvante no tratamento do câncer. Nesse sentido, fica como lacuna a comprovação empírica em outros diagnósticos oncológicos, necessitando novos estudos para avaliar a eficácia desses modelos de gerenciamento para os demais tipos de câncer.

\section{Referências}

ABDOLLAHI, F.; KHAN, M. S. Role of Mindfulness-Based Cognitive Therapy in Alleviating Psychological Distress among Cancer Patients. The International Journal of Indian Psychology, Gujarat, v. 2, n. 1, p. 108-120, Oct./ Dec. 2014.

ANDERSEN, B, L. et al. Stress and immune responses after surgical treatment for regional breast cancer. Journal of the National Cancer Institute, Bethesda, v. 90, n. 1, p. 30-36, Jan. 1998. doi: 10.1093/jnci/90.1.30. 
ANDERSEN, B. L.; KIECOLT-GLASER, J. K.; GLASER, R. A biobehavioral model of cancer stress and disease course. American Psychologist, Washington, v. 49, n. 5, p. 389-404, May 1994. doi: 10.1037/0003-066X.49.5.389.

ANTONI, M. H. et al. Cognitive-behavioral stress management intervention decreases the prevalence of depression and enhances benefit finding among women under treatment for early-stage breast cancer. Health Psychology, Washington, v. 20, n. 1, p. 20-32, jan. 2001. doi: 10.1037/0278-6133.20.1.20.

ANTONI, M. H. et al. Cognitive-behavioral stress management reverses anxiety-related leukocyte transcriptional dynamics. Biological Psychiatry, New York, v. 71, n. 4, p. 366-372, Feb. 2012. doi: 10.1016/j.biopsych.2011.10.007.

ARMAIZ-PENA, G. N. et al. Neuroendocrine influences on cancer progression. Brain, Behavior, and Immunity, Amsterdam, v. 30, n. suppl., p. S19-S25, Mar. 2013. doi: 10.1016/j.bbi.2012.06.005.

BROTHERS, B. M. et al. Cancer patients with major depressive disorder: testing a biobehavioral/cognitive behavior intervention. Journal of Consulting and Clinical Psychology, Washington, v. 79, n. 2, p. 253-260, Apr. 2011. doi: $10.1037 / \mathrm{a} 0022566$.

BERGNER, M. et al. The Sickness Impact Profile: development and final revision of a health status measure. Medical Care, Hagerstown, v. 19, n. 8, p. 787-805, Aug. 1981.

DEROGATIS, L. R. Derogatis affect balance scale. 1975. Disponível em: <http://www.statisticssolutions.com/derogatis-affect-balance-scale-dabs/>. Acesso em: 20 nov. 2016.

BRADY, M. J. et al. Reliability and validity of the Functional Assessment of Cancer Therapy-Breast quality-of-life instrument. Journal of Clinical Oncology, Alexandria, v. 15, n. 3, p. 974-986, Mar. 1997. doi: 10.1200/ JCO.1997.15.3.974.

COSTANZO, E. S.; SOOD, A. K.; LUTGENDORF, S. K. Biobehavioral influences on cancer progression. Immunology and Allergy Clinics of North America, Philadelphia, v. 31, n. 1, p. 109-132, Feb. 2011. doi: 10.1016/j. iac.2010.09.001.
CUTRONA, C. E.; RUSSELL, D. W. The provisions of social relationships and adaptation to stress. In: JONES, W. H.; PERLMAN, D. Advances in Personal Relationships, Greenwitch: JAI Press, 1987. p. 37-67.

GARLAND, S. N. et al. Mindfulness-based stress reduction compared with cognitive behavioral therapy for the treatment of insomnia comorbid with cancer: a randomized, partially blinded, noninferiority trial. Journal of Clinical Oncology, Alexandria, v. 32, n. 5, p. 449-457, Feb. 2014. doi: 10.1200/JCO.2012.47.7265.

GARSSEN, B.; BOOMSMA, M. F.; BEELEN, R. H. J. Psychological factors in immunomodulation induced by cancer surgery: a review. Biological Psychology, Amsterdam, v. 85, n. 1, p. 1-13, Sep. 2010. doi: 10.1016/j.biopsycho.2010.05.006.

GUDENKAUF, L. M. et al. Brief cognitive-behavioral and relaxation training interventions for breast cancer: a randomized controlled trial. Journal of Consulting and Clinical Psychology, Washington, v. 83, n. 4, p. 677-688, 2015. doi: $10.1037 /$ ccp0000020.

HENDERSON, V. P. et al. The effects of mindfulness-based stress reduction on psychosocial outcomes and quality of life in early-stage breast cancer patients: a randomized trial. Breast Cancer Research and Treatment, Dordrecht, v. 131, n. 1, p. 99-109, Jan. 2012. doi: 10.1007/s10549-011-1738-1.

HINAMI, K. et al. Health perceptions and symptom burden in primary care: measuring health using audio computer-assisted self-interviews. Quality of Life Research, Oxford, v. 24, n. 7, p. 1575-1583, Jul. 2015. doi: 10.1007/ s11136-014-0884-4.

INSTITUTO NACIONAL DO CÂNCER. Estimativa 2016-2017: incidência de câncer no Brasil. 2016. Disponível em: <http://mortalidade.inca.gov.br/Mortalidade/>. Acesso em: 01 dez. 2016.

JADAD, A. R. et al. Assessing the quality of reports of randomized clinical trials: is blinding necessary? Controlled Clinical Trials, New York, v. 17, n. 1, p. 1-12, Feb. 1996. doi: 10.1016/0197-2456(95)00134-4. 
LECHNER, S. C. et al. Does a community-based stress management intervention affect psychological adaptation among underserved black breast cancer survivors? Journal of the National Cancer Institute Monographs, Bethesda, v. 2014, n. 50, p. 315-322, Nov. 2014. doi: 10.1093/jncimonographs/lgu032.

ROBINS, J. L. W. et al. Psychoneuroimmunology-based stress management during adjuvant chemotherapy for early breast cancer. Evidence-Based Complementary and Alternative Medicine, United Kingdom, v. 2013, p. 1-7, 2013. doi: 10.1155/2013/372908.

STAGL, J. M. et al. A randomized controlled trial of cognitive-behavioral stress management in breast cancer: survival and recurrence at 11-year follow-up. Breast Cancer Research and Treatment, Dordrecht, v. 154, n. 2, p. 319-328, 2015. doi: 10.1007/s10549-015-3626-6.
TRAEGER, L. et al. Identifying how and for whom cognitive-behavioral stress management improves emotional well-being among recent prostate cancer survivors. Psychooncology, Chichester, v. 22, n. 2, p. 250-259, Feb. 2013. doi: 10.1002/pon.2074.

WEISS, D. S. The impact of event scale: revised. In: WILSON, J. P.; SO-KUM TANG, C. C. Cross-cultural assessment of psychological trauma and PTSD. New York, Springer, 2007. p. 219-238.

VANDENBERGHE, L.; SOUSA, A. C. A. Mindfulness nas terapias cognitivas e comportamentais. Revista Brasileira de Terapias Cognitivas, Rio de Janeiro, v. 2, n. 1, p. 35-44, jun. 2006.

VARGAS, S. et al. Sleep quality and fatigue after a stress management intervention for women with early-stage breast cancer in Southern Florida. International Journal of Behavioral Medicine, London, v. 21, n. 6, p. 971-981, dec. 2014. doi: 10.1007/s12529-013-9374-2. 